\title{
WaFIRS: a waveguide far-IR spectrometer: enabling spectroscopy of high-z galaxies in the far-IR and submillimeter
}

Charles M. Bradford, Bret J. Naylor, Jonas Zmuidzinas, James J. Bock, J. Gromke, et al.

Charles M. Bradford, Bret J. Naylor, Jonas Zmuidzinas, James J. Bock, J. Gromke, Hien Nguyen, Mark Dragovan, Minhee Yun, Lieko Earle, Jason Glenn, Hideo Matsuhara, Peter A. R. Ade, Lionel Duband, "WaFIRS: a waveguide far-IR spectrometer: enabling spectroscopy of high-z galaxies in the far-IR and submillimeter," Proc. SPIE 4850, IR Space Telescopes and Instruments, (5 March 2003); doi: 10.1117/12.461572

Event: Astronomical Telescopes and Instrumentation, 2002, Waikoloa, Hawai'i, United States 


\title{
WaFIRS, a waveguide far-IR spectrometer: enabling spectroscopy of high-z galaxies in the far-IR and submillimeter
}

\author{
C.M. Bradford ${ }^{a}$, B.J. Naylor ${ }^{a}$ J. Zmuidzinas ${ }^{a}$ J.J. Bock $^{a b}$, J. Gromke ${ }^{a}$ \\ H. Nguyen ${ }^{b}$, M. Dragovan ${ }^{b}$, M. Yun ${ }^{b}$ L. Earle ${ }^{c}$, J. Glenn ${ }^{c}$, \\ H. Matsuhara ${ }^{d}$, P.A.R. Ade $^{e}$, L. Duband $f$ \\ ${ }^{a}$ California Institute of Technology, Pasadena, CA \\ ${ }^{b}$ Jet Propulsion Laboratory, Pasadena, CA \\ ${ }^{c}$ CASA, University of Colorado, Boulder, CO \\ ${ }^{d}$ ISAS, Sagamihara, Japan \\ eUniversity of Wales, Cardiff, UK \\ ${ }^{f} \mathrm{CEA}$, Grenoble, France
}

\begin{abstract}
The discovery of galaxies beyond $\mathrm{z} \sim 1$ which emit the bulk of their luminosity at long wavelengths has demonstrated the need for high-sensitivity, broad-band spectroscopy in the far-IR/submm/mm bands. Because many of these sources are not detectable in the optical, long-wavelength spectroscopy is key to measuring their redshifts and ISM conditions. The continuum source list will increase in the coming decade with new ground-based instruments (SCUBA2, Bolocam, MAMBO), and the surveys of HSO and SIRTF. Yet the planned spectroscopic capabilities lag behind, in part due to the difficulty in scaling existing IR spectrograph designs to longer wavelengths. To overcome these limitations, we are developing WaFIRS, a novel concept for long-wavelength spectroscopy which utilizes a parallel-plate waveguide and a curved diffraction grating. WaFIRS provides the large $(\sim 60 \%)$ instantaneous bandwidth and high throughput of a conventional grating system, but offers a dramatic reduction in volume and mass. WaFIRS requires no space overheads for extra optical elements beyond the diffraction grating itself, and is two-dimensional because the propagation is confined between two parallel plates. Thus several modules could be stacked to multiplex either spatially or in different frequency bands. The size and mass savings provide opportunities for spectroscopy from space-borne observatories which would be impractical with traditional spectrographs. With background-limited detectors and a cooled $3.5 \mathrm{~m}$ telescope, the line sensitivity would be comparable to that of ALMA, with instantaneous broad-band coverage. We present the spectrometer concept, performance verification with a mm-wave prototype, and our progress toward a cryogenic astronomical instrument.
\end{abstract}

Keywords: far-IR, spectroscopy, waveguide, grating, planar spectrometer

\section{INTRODUCTION}

The next decade will see a marked change in our understanding of the far-IR and submillimeter universe. The Space Infrared Telescope Facility (SIRTF) and Herschel Space Observatory (HSO) missions will map the continuum to their confusion limits, making progress toward resolving the IR / submm background into its discrete sources: star-forming and AGN galaxies at moderate and high redshift. However, continuum source counts represents only the first step. Measuring the redshifts and physical conditions in these sources will require spectroscopy. Such studies will be difficult with the spectrometers on HSO and SIRTF at the highest redshifts, due to limited sensitivity and/or limited bandwidth at far-IR wavelengths. Relative to planned farinfrared and submillimeter platforms, there are still several orders of magnitude of improvement possible before

Further author information: Send correspondence to C.M.B: E-mail: bradford@caltech.edu, tel: 16263954601 
the encountering the fundamental limits set by the natural backgrounds. Key technical challenges include the sensitivity of long-wavelength detectors, and the size of existing spectrometer modules when scaled to these wavelengths. To overcome the size issue, we are developing a new technology for a compact, broad-band spectrometer for the far-IR and submillimeter. WaFIRS, the Waveguide Far-IR Spectrometer consists of a curved diffraction grating, entrance feed horn and detector feed horns all inside a two-dimensional parallel plate propagation medium. The compact size facilitates broad-band spectroscopy from space which is not possible with conventional spectrometer designs. After a review of the scientific motivation, we describe our spectrometer development effort, testing of the first prototype, and progress toward a cryogenic astronomical test bed.

\section{SCIENTIFIC MOTIVATION FOR FAR-IR SPECTROSCOPY}

The advent of large-format bolometer arrays for wavelengths around $1 \mathrm{~mm}$ (SCUBA, MAMBO) has revealed a new class of galaxies which are likely at medium to high redshift. These sources are cosmologically significant their counts reproduce much of the diffuse far-IR / submillimeter background radiation, representing the energy generated by all galaxies over the history of the universe ${ }^{1,2}$. These submillimeter galaxies are luminous systems similar to the nearby IR galaxies discovered with IRAS. Of the nearly 200 submillimeter galaxies discovered thus far, only a fraction have confirmed spectroscopic redshifts and well-determined properties at other wavelengths. This is because the sources are very dusty with high extinction at short wavelengths, the optical and UV energy is almost entirely reprocessed and reradiated between $\lambda=50 \mu \mathrm{m}$ and $1 \mathrm{~mm}$, making the optical counterparts very faint. While there are spectral features that could be used in the millimeter / submillimeter, the instantaneous bandwidth of heterodyne millimeter-wave receivers is currently a small fraction of unity, so searching for lines in sources with unknown redshifts is impractical.

The long-wavelength continuum source list will only increase in the next decade with new ground-based instruments (SCUBA2, Bolocam) and the confusion-limited surveys of HSO and SIRTF. Long wavelength spectroscopy with large instantaneous bandwidth is the key to measuring these sources' redshifts, which constrain their luminosities, sizes, and masses. Moreover, the wide variety of spectral features in the mid- and far-IR provide information on the conditions in all phases of the interstellar medium, ionized, neutral atomic, and molecular (see Table 1). We highlight some of the key spectroscopic probes.

Fine-structure transitions of abundant elements, both neutral and ionized have luminosities up to a few $\times 10^{-3}$ of the total bolometric luminosity. These lines measure the gas conditions and UV field properties in regions where stellar or AGN luminosity is input into the ISM. Mid- and far-IR fine structure lines have been used to study the starburst conditions in nearby galaxies, ${ }^{3,5,6,7}$ and recently, as an AGN / starburst discriminator in ULIGs ${ }^{8}$. Molecular rotational transitions such as the rotational transitions of $\mathrm{CO}$ are the dominant coolant of molecular gas and our primary probe of its temperature and density. Lower-J CO transitions are important for cooling quiescent molecular gas heated to a few $10 \mathrm{~K}$ by cosmic rays, while mid- and high-J CO lines trace warm gas associated with UV and shock heating near star-formation sites ${ }^{9,10,11}$. Other abundant species such as $\mathrm{OH}$ and $\mathrm{CH}$ constrain molecular gas column densities and abundances through absorption transitions ${ }^{12,13}$. This suite of molecular lines also has potential as a redshift probe for extremely obscured sources (those weak in fine structure lines) such as Arp 220. The rotational lines of $\mathrm{H}_{2}$ are likely to be the primary way for metal-free clouds to cool and collapse to form the very first stars at redshifts between 10 and $50^{14,15}$. At the redshifts of formation, the lines are shifted to the far-IR and submillimeter, making it a powerful regime to observe the first collapsing objects in the universe. Finally, where large molecules overlap with dust grains, polycyclic aromatic hydrocarbons (PAHs) are an outstanding probe of starburst activity. These mid-IR bands are very prominent in starburst systems, the brightest emitting as much as $1-4 \%$ of the bolometric luminosity due to their broad widths ${ }^{16}$. They will make an excellent high-sensitivity redshift probe.

Unfortunately, though there are a wealth of diagnostics for dusty galaxies in the far-IR and submillimeter, the wavelength range is not generally accessible from the ground. The atmospheric windows shortward of $\lambda \sim 700 \mu \mathrm{m}$ are accessible only occasionally from mountaintop sites. Between 40 and $200 \mu \mathrm{m}$, the atmosphere is completely opaque from all terrestrial sites. While there are space missions planned to observe these wavelengths in the continuum (SIRTF, HSO), and these missions include spectroscopic capabilities, there is a large gap between the spectral capabilities of planned missions, and the fundamental limits achievable with even existing detector 
Table 1. Far-IR Spectral Features

\begin{tabular}{|c|c|c|c|c|c|}
\hline Species & Wavelength $[\mu m]$ & $f_{\mathrm{M} 82}$ & $f_{\text {Arp220 }}$ & Diagnostic Utility & refs \\
\hline \multicolumn{6}{|c|}{ Ionized Gas Fine Structure Lines } \\
\hline O IV & $25.9,54.9$ & & \multirow{4}{*}{$7.5 \times 10^{-5}$} & \multicolumn{2}{|l|}{ Primarily AGN } \\
\hline S IV & 10.5 & $2.1 \times 10^{-5}$ & & Probes gas density and & 17 \\
\hline $\mathrm{Ne}$ II & 12.3 & $1.2 \times 10^{-3}$ & & UV field hardness in & 17,8 \\
\hline Ne III & $15.6,36.0$ & $2.05 \times 10^{-4}$ & & star formation HII & 17 \\
\hline S III & $18.7,33.5$ & $1.0 \times 10^{-3}$ & \multirow{4}{*}{$7.3 \times 10^{-5}$} & regions. & 17 \\
\hline Ar III & 21.83 & $9.1 \times 10^{-6}$ & & & 17 \\
\hline O III & $51.8,88.4$ & $1.3 \times 10^{-3}$ & & & 5 \\
\hline $\mathrm{N}$ III & 57.3 & $4.2 \times 10^{-4}$ & & & 5 \\
\hline $\mathrm{N}$ II & 122,205 & $2.1 \times 10^{-4}$ & & Diffuse HII regions & 5 \\
\hline \multicolumn{6}{|c|}{ Neutral Gas Fine Structure Lines } \\
\hline Si II & 34.8 & $1.1 \times 10^{-3}$ & $7.7 \times 10^{-5}$ & \multirow{4}{*}{$\begin{array}{l}\text { Density and temperature probes } \\
\text { of photodissociated-neutral } \\
\text { gas interface between HII } \\
\text { regions and molecular clouds. }\end{array}$} & 17,8 \\
\hline O I & $63.1,145$ & $2.2 \times 10^{-3}$ & $-6.8 \times 10^{-5}$ & & 5,13 \\
\hline C II & 158 & $1.6 \times 10^{-3}$ & $1.3 \times 10^{-4}$ & & 5,18 \\
\hline $\mathrm{C} \mathrm{I}$ & 370 & $6.2 \times 10^{-6}$ & $1.2 \times 10^{-5}$ & & 19,20 \\
\hline \multicolumn{6}{|c|}{ Molecular Lines } \\
\hline $\mathrm{H}_{2}$ & $9.6612 .3,17.0,28.2$ & $2 \times 10^{-5}$ & $3 \times 10^{-5}$ & Coolants of first collapse & 17 \\
\hline $\mathrm{CH}$ & 149 & & $-4 \times 10^{-5}$ & Ground state absorption, & 13 \\
\hline $\mathrm{OH}$ & $34.6,53.3,79.1,119$ & $-2 \times 10^{-6}$ & $-2 \times 10^{-4}$ & gives column and abundance. & 5,13 \\
\hline $\mathrm{OH}$ & $98.7,163$ & & $5 \times 10^{-5}$ & Emission & 13 \\
\hline $\mathrm{H}_{2} \mathrm{O}$ & $73.5,90,101,107,180$ & & $\pm 5 \times 10^{-5}$ & & 13 \\
\hline $\mathrm{CO}$ & $325,372,434,520$ & $3 \times 10^{-6}$ & $\left(1 \times 10^{-5}\right)$ & Mid-J, $50 \mathrm{~K}<\mathrm{T}<200 \mathrm{~K}$ mol. gas & 21 \\
\hline
\end{tabular}

Notes: The $f_{\mathrm{M} 82}$ and $f_{\mathrm{Arp} 220}$ are the ratios of the line luminosity to the total bolometric luminosity. M82 is an example of a starburst galaxy bright in most the fine structure lines, whereas Arp220 is more highly obscured and weaker in the short-wavelength atomic lines, but shows strong molecular lines features. AGN sources, such as NCG 1068 and Mrk 231 have bright emission in the OIV lines. Most of the mid-J CO lines fall between ISO and the ground-based windows, and the parentheses represent expected fluxes for Arp 220. Based on accessible transitions $(\mathrm{J}=7-6,6-5)$ observed in the nearby starburst galaxies, and a handful of observations of $\mathrm{CO}$ in quasars and submillimeter galaxies at high redshift any dusty galaxy should show bright emission from the CO lines up to at least $\mathrm{J}=8-7(325 \mu \mathrm{m})$.

technology. For example, the sensitivity attainable from a modest $(3.5 \mathrm{~m})$ cool $(\mathrm{T}<15 \mathrm{~K})$ space telescope is much better than what is currently planned between SIRTF and ALMA (see Figure 1). The effective line survey speed, proportional to the inverse of the sensitivity squared divided by the instantaneous bandwidth, shows a more dramatic gain of several orders of magnitude (also in Figure 1). These large potential gains are possible in part because the raw sensitivity improves substantially by cooling the telescope to $15 \mathrm{~K}$ or lower (HSO is expected to operate at about $60 \mathrm{~K}$ ).

\section{SUITABILITY OF CONVENTIONAL SPECTROMETER MODULES}

While there are a variety of options for a far-IR and submillimeter spectrometer, some are better suited to the science goals outlined above - observing high-redshift dusty galaxies with high sensitivity. Heterodyne spectrometers will always be the instruments of choice for the highest spectral resolutions and are required for phase preservation in interferometric experiments. However, they are not well-suited to sensitive extragalactic observations because of comparatively small bandwidth, and a quantum noise which results in a sensitivity 

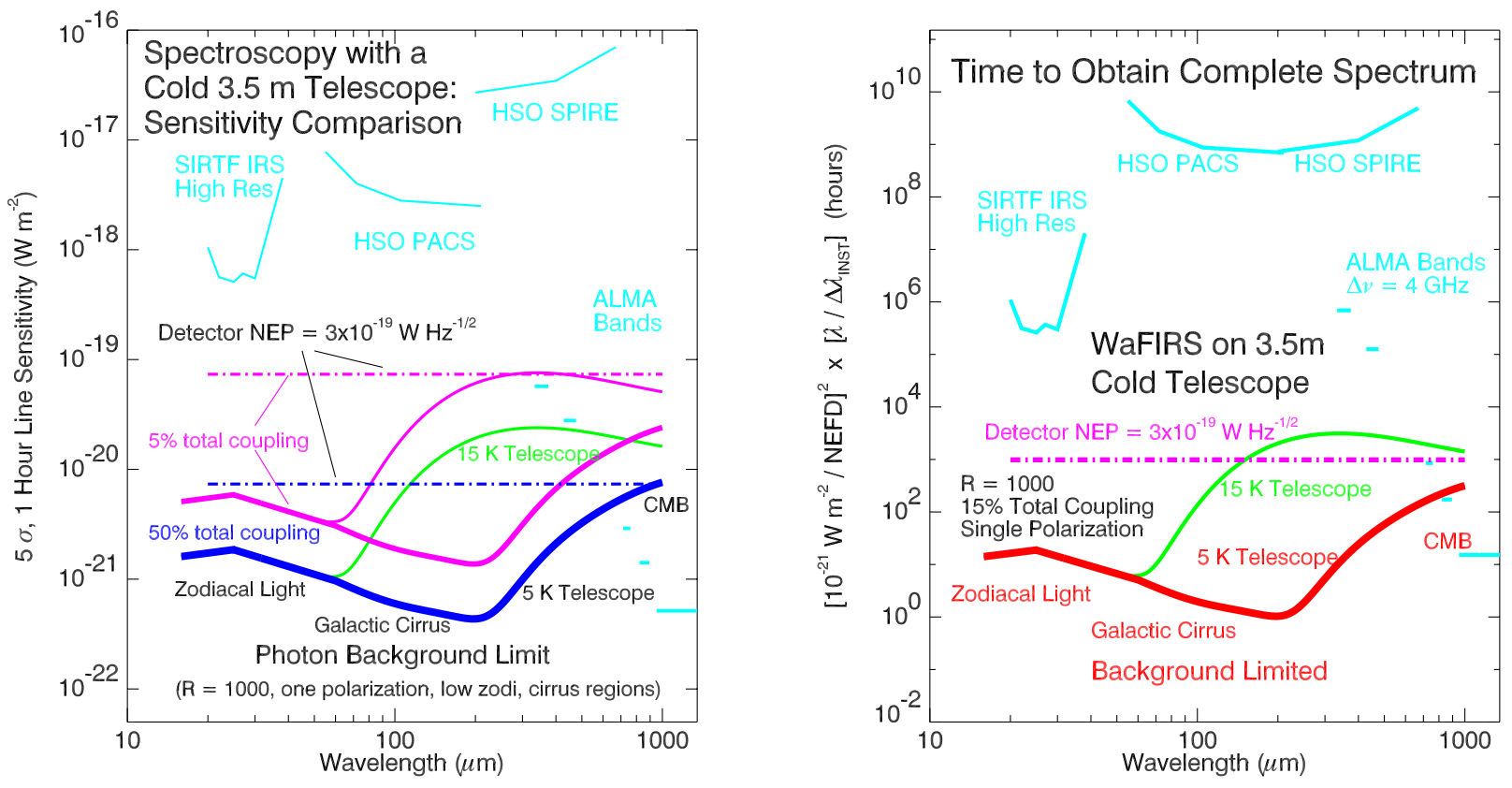

Figure 1. [Left] Spectral sensitivity on a cold $3.5 \mathrm{~m}$ space telescope. The $5 \%$ and $50 \%$ total couplings are conservative bounds to what might be achieved with a real spectrometer. Background noise is calculated from the fluctuations in the zodiacal and cirrus backgrounds, toward patches of low intensity. ${ }^{22}$ If historical trends continue, background-limited performance at these frequencies will be possible, and gains of 2-4 orders of magnitude in raw sensitivity can be achieved. [Right] Sensitivity calculations, but including the effects of instrumental bandwidth for obtaining a complete spectrum. Under reasonable assumptions ( $15 \%$ efficiency, one polarization only), a broad-band spectrometer on a cold $3.5 \mathrm{~m}$ telescope will have line survey speeds 6 orders of magnitude faster than the spectrometers on HSO, making far-IR line surveys practical. Such an instrument would be faster than ALMA for $\lambda<600 \mu \mathrm{m}$ for point-source line surveys. (ALMA will provide instantaneous mapping and velocity relocity resolution, and is complementary to redshift determination.) For a 10-meter class cold telescope such as SAFIR, the sensitivity advantages are improved an additional factor of more than 5 beyond what is plotted.

penalty of order

$$
\mathrm{NEP}_{\text {quantum }} \sim \mathrm{h} \nu \sqrt{\Delta \nu}
$$

In contrast, a direct-detection spectrometer can approach the noise due to background fluctuations alone:

$$
\mathrm{NEP}_{\mathrm{BG}}=\mathrm{h} \nu \sqrt{\Delta \nu} \sqrt{\overline{\mathrm{n}}(\overline{\mathrm{n}}+1)} .
$$

Here, $\overline{\mathrm{n}}=\eta(\exp (\mathrm{h} \nu / \mathrm{kT})-1)^{-1}$ is the reduced photon occupation number which become exponentially small for $\mathrm{h} \nu>\mathrm{kT}$, and illustrates the advantage of a cold telescope. At $\lambda=300 \mu \mathrm{m}$, on a $10 \mathrm{~K}$ telescope with $5 \%$ coupling, $\overline{\mathrm{n}}=4 \times 10^{-4}$, and the quantum noise is a factor of $1 / \sqrt{\overline{\mathrm{n}}}=50$ larger than the background limit achievable with a direct-detection instrument.

Because sources will be taken from preceding continuum surveys and will be spatially unresolved with the telescopes under consideration, an imaging spectrometer is not a high priority. On the other hand, the redshifts may not be known and the diagnostic lines are distributed over a broad spectral range, so a large instantaneous spectral bandwidth is critical. Given that in the far-IR and submillimeter, the total number of detectors is typically a constraint, it therefore desirable to have the detectors arrayed spectrally rather than spatially, and an imaging monochromator such as a Fabry-Perot is not the instrument of choice. For ultimate sensitivity, a Fourier transform spectrometer (FTS) is not ideal because it places the entire spectral bandwidth and its associated photon noise onto a single detector. An FTS is appropriate only when using a detector which is not background-limited at the spectrometer resolution. 


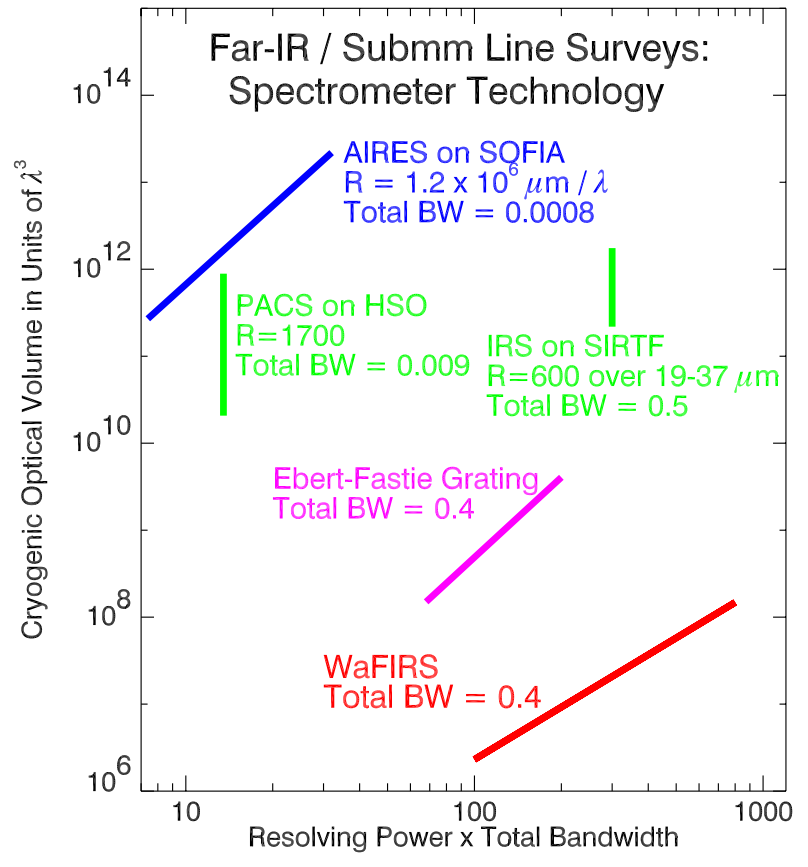

Figure 2. Sizes of existing spectrometer modules compared with their survey capability. The spectral survey capability is given by the product of resolving power (up to $\mathrm{R} \sim 1000$ ) and total bandwidth. Modest resolution, broad bandwidth systems exist (e.g. the SIRTF IRS modules), but are prohibitively large when scaled to far-IR and submillimeter wavelengths. WaFIRS offers a compact broad-band system for longer wavelengths. We point out that for line mapping, the PACS and AIRES enclosures provide multiple beams of spatial coverage, not provided in a single WaFIRS module.

The obvious choice for background-limited point-source spectroscopy is a diffraction grating. Gratings have been used in astronomy for decades, and recently in the Infrared Space Observatory (ISO) as cryogenic, space-borne infrared spectrometers. When operated in first order, a grating naturally provides an octave of instantaneous bandwidth, and the resolution can be increased by increasing the grating size, roughly $\mathrm{d} \sim \lambda \times \mathrm{R} / 2$. At far-IR and submillimeter wavelengths, this size quickly becomes prohibitively large, particularly since real instruments are typically larger than the fundamental limit because they include collimating and imaging mirrors as well as order-sorting elements. For example, each of the spectrometer modules on SIRTF, measures about $40 \times 15 \times 20 \mathrm{~cm}$, with a maximum $\lambda \times \mathrm{R}$ product of $2 \mathrm{~cm}(\mathrm{R}=600$ at $37 \mu \mathrm{m})$. To scale such an instrument up for a wavelength of $200 \mu \mathrm{m}$ would result in a long dimension of over 2 meters, prohibitive for a space mission. Another example is the PACs spectrometer for HSO, an image slicing spectrometer which provides $\mathrm{R}=1500$ out to $200 \mu \mathrm{m}(\lambda \times \mathrm{R}=30 \mathrm{~cm})$. The size of the cryogenic enclosure is quite large, roughly $80 \mathrm{~cm} \times 80 \mathrm{~cm} \times 30 \mathrm{~cm}$, and because the instrument is designed for imaging spectroscopy, it only provides 16 spectral resolution elements, or $1 \%$ instantaneous bandwidth. The sizes of existing spectrometers are shown in Figure 2 in units of $\lambda^{3}$, plotted against the total number of spectral resolution elements.

\section{WAFIRS DESIGN DETAILS}

At long wavelengths, spectrometer space is at a premium, and a spectrometer architecture should make efficient use of the grating length as interference path. This means that the angle between the grating normal and the incident radiation should be large, of order 1 radian. To operate over a broad band, the diffracted angle then needs to vary from a value comparable to the incident angle (to accommodate $\lambda_{\max }$ ), to near normal (to accommodate $\lambda_{\max } / 2$ ). The combination of these fundamental requirements with an optical system (which requires fast curvature in the cross dispersion dimension to focus the light) is a difficult design. On the other hand, eliminating the second dimension dramatically simplifies the optical requirements. Furthermore, proper use of a curved grating allows a single powered surface to simultaneously disperse and focus the radiation, resulting in a space efficient arrangement. WaFIRS is conceptually similar to the slit spectrometers with curved gratings used by Rowland ${ }^{23}$, Eagle, Wadsworth and others ${ }^{24}$. Though WaFIRS uses the same basic layout, it is based on propagation of a single EM mode in a two-dimensional medium bounded by parallel, conducting plates. We outline the details of the spectrometer design, beginning with the key aspects of propagation in 
parallel plate waveguide.

\subsection{Parallel Plate Propagation Medium}

WaFIRS uses a $\mathrm{TE}_{1}$ propagation mode in parallel plate waveguide. In addition to offering the lowest propagation loss of the parallel-plate modes, the $\mathrm{TE}_{1}$ field profile is identical to the $\mathrm{TE}_{10}$ profile in rectangular waveguide,

$$
E_{y}=E_{y, 0} \sin (\pi x / d) e^{-j \beta z}
$$

where $d$ is the plate separation distance, $x$ refers to this dimension, and $z$ refers to the propagation direction. Thus rectangular waveguide can readily be used as an input feed, convenient because it can also be coupled to other geometries such as circular feed horns matched to a telescope focus. The dispersion is that of waveguide, the wavelength in the medium is given by ${ }^{25}$

$$
\lambda_{G}=2 \pi\left(k^{2}-k_{c}^{2}\right)^{-1 / 2}=\left(\frac{1}{\lambda_{0}^{2}}-\frac{1}{4 d^{2}}\right)^{-\frac{1}{2}},
$$

where the plate spacing determines the cutoff frequency $\nu_{c}=c / 2 d$. Below this frequency, propagation is not possible, and as the cutoff frequency is approached, the dispersion relation becomes very sharp with $\lambda_{G}$ diverging. Thus geometrically, it is inconvenient to operate near cutoff. The propagation loss due to currents in the imperfectly-conducting plates also diverges near cutoff. The absorption coefficient can be expressed as

$$
\alpha\left[\mathrm{cm}^{-1}\right]=\frac{\lambda_{G} \lambda_{0}}{2 d^{3}} \frac{R_{S}}{\eta},
$$

where $R_{S}$ and $\eta$ are the surface resistance of the plates and the impedance of free space, respectively. For frequencies well above cutoff, $\lambda_{G} \sim c / \nu$, and this goes as $d^{-3}$. Thus for a given frequency and value of $R_{S}$, increasing the plate separation can make the propagation loss negligible compared with the other losses in the system. To make an estimate, consider that the propagation distance in the spectrometer is $\sim 2 \times$ the resolving power $\mathbf{R}$ in wavelength units. Thus it is desired that the product $2 \lambda_{G} \alpha \mathbf{R}$ is much less than unity. For a good conductor at room temperature, $R_{S} \sim 0.2 \Omega$, and the requirement can be expressed as

$$
d \gg 8 \times 10^{-2}\left(\lambda_{G}^{2} \lambda \mathbf{R}\right)^{1 / 3} .
$$

Of course, operation at cryogenic frequencies substantially decreases the surface resistance, making the above expression less stringent. However, the tolerances on surface smoothness (also to avoid loss), and flatness (to maintain a uniform dispersion relation everywhere in the region) each have a similar decrease with separation distance. At some point, larger spacing becomes detrimental - since ultimately the feeds and detectors must have sizes of order the wavelength, and the transformation from this small size to a much larger plate separation carries the risk of loss to the many other modes which become viable at large spacing. For these practical reasons, the spectrometer should have a plate separation a few times greater than the cutoff spacing.

\subsection{Curved Grating Architecture}

The spectrometer design begins with a Rowland Circle construction ${ }^{23}$ subject to the fundamental geometric ideas outlined above for space utilization and broad bandwidth. A sketch of the concept is shown in Figure 3 . In this geometry, the input feed and the focal positions lie on the same circular arc; the grating arc is tangent with twice the radius. The basic layout is fixed by three choices: the size of the Rowland circle, the location of the illuminating horn on the circle, and the position of the focus for a given frequency on the same circle. In recent years, spectrometers have been developed for optical wavelengths with flexibility in the facet positioning. It has been pointed out that each facet can be individually positioned, its two degrees of freedom constrained with two equations ${ }^{26}$. In our system, to provide good performance over a broad band, we choose two stigmatic frequencies on either edge of the band for which the grating provides aberration-free performance. The constraints are:

$$
\begin{aligned}
& 0=\epsilon_{i, 1}=R_{0, i}\left(x_{i}, y_{i}\right)+R_{1, i}\left(x_{i}, y_{i}\right)+i \lambda_{1}-R_{0}-R_{1} \\
& 0=\epsilon_{i, 2}=R_{0, i}\left(x_{i}, y_{i}\right)+R_{2, i}\left(x_{i}, y_{i}\right)+i \lambda_{2}-R_{0}-R_{2}
\end{aligned}
$$




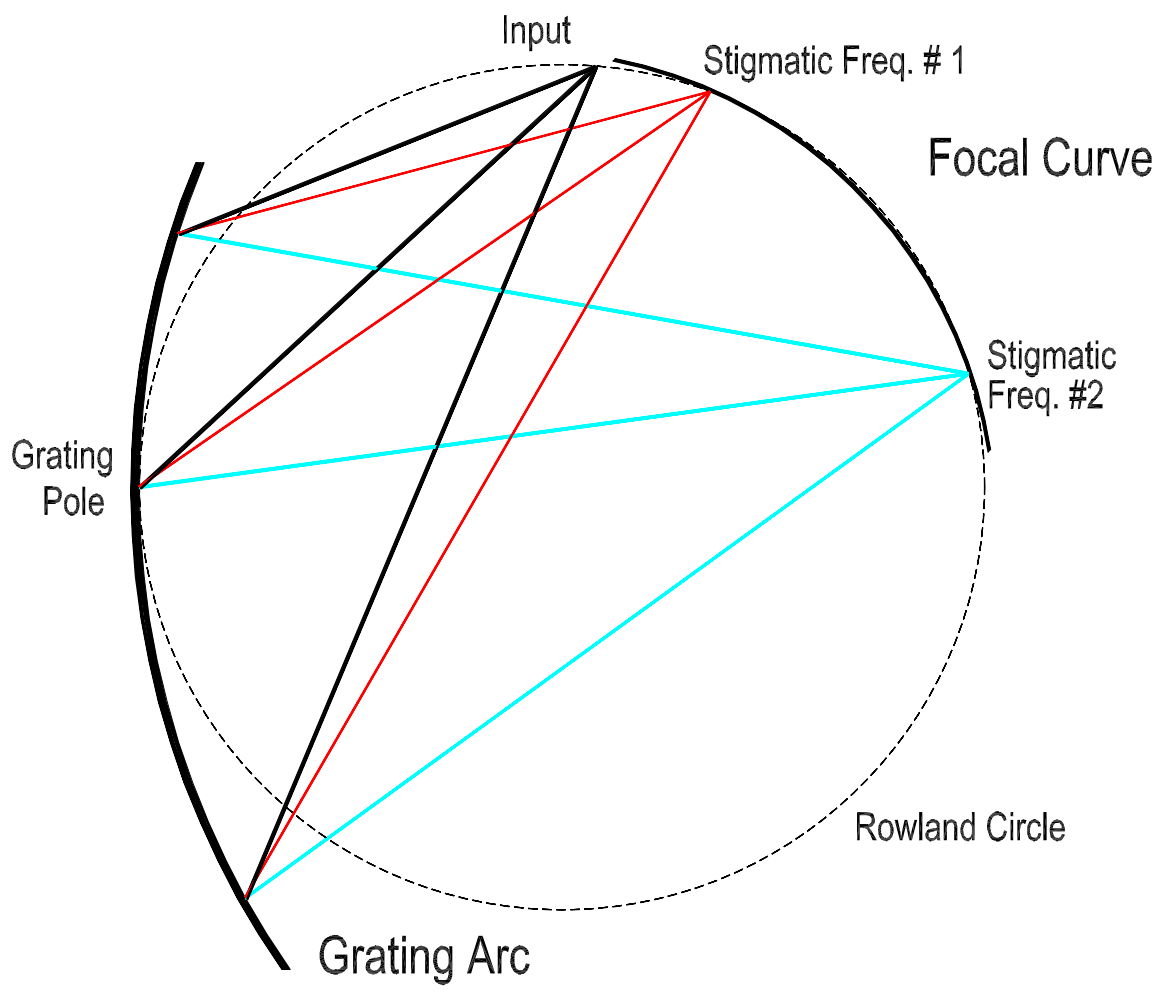

Figure 3. Spectrometer Geometry. WaFIRS utilizes a basic architecture similar to some of the first grating spectrometers implemented by H.A. Rowland in the late 19th century. Facets ruled on the grating arc both diffract and focus the radiation to locations on focal curve. Key features improve the concept for modern applications. For WaFIRS, each facet is individually positioned to provide perfect performance at the two stigmatic frequencies. This allows the grating arc to subtend an angle $\sim 1$ radian, and the length of the arc is comparable to the largest dimension in the system, giving the longest interference path possible. Also, eliminating the second dimension by confining the propagation makes for easy and efficient coupling to detectors.

where $i$ is the facet index and $1 \& 2$ refer to the two stigmatic frequencies. $R_{0, i}$ is the distance from the input position to facet $i$, and $R_{1, i}\left(R_{2, i}\right)$ is the distance from facet $i$ to the focus position for frequency 1 (2). The $R_{0}$, $R_{1}, R_{2}$ are the distances where the facet index is 0 , referring to the pole of the grating where the grating arc intersects the Rowland circle. They are therefore constants determined by the initial design choices. The effect of the constraint equations is to make the propagation phase from the input to a facet to the output change by exactly $2 \pi$ from one facet to the next. Because the partial derivatives of $\epsilon$ are calculable, we use Newton's method to iteratively solve for $x_{i}, y_{i}$ using Rowland's original facet positions as initial guesses. The resulting arc has slightly more curvature than Rowland's grating, and a facet spacing increasing away from the pole.

The total number of facets is limited by the space available. We have found that the most efficient system places the majority of the facets on the side opposite the input position as shown in Figure 3. The illumination of the grating is determined by the size and orientation of the input waveguide. The choice is a compromise between increasing resolving power with a broader illumination pattern and minimizing spillover loss beyond the edges of the grating. In situations where the detectors are background-limited and the photon occupation number is large (e.g. at millimeter wavelengths), the compromise can be shifted in favor of spectral resolution, because there is only a small sensitivity penalty for decreased throughput.

To couple the radiation to detectors, the ideal arrangement is a close-packed linear array of bare detectors each coupling to all angles. Although there is only a single radiation mode in the plate separation (vertical) direction, the guide is overmoded, and a single line antenna in the center of the plates will scatter into higherorder vertical modes instead of absorbing efficiently. In the horizontal dimension, the incident radiation is 
analogous to a diffraction-limited beam with fast $f /$, and is it desired to couple as many modes as possible to absorb the most power incident from the grating, especially for photons incident between two detectors. A further complication is that for most of the band, the direction normal to the focal curve (radial) is not optimally oriented toward the grating (refer to Figure 3). The deviation can be as much as $35^{\circ}$ at the lowest frequencies. This also argues for coupling as many modes as possible and for carefully orienting the detectors as a function of frequency. For traditional individual bolometric detectors which require space overheads beyond the absorber, the radiation must be either concentrated with some tapered feed for each detector (analogous to feed horns used in a 2-D spatial array), or rerouted from the focal curve to a location which can accommodate the absorber and its overhead. A scheme in which the radiation for every other detector is routed above or below the focal curve offers the advantage that the feed need not taper in width and therefore does not reflect higher-order modes which could be excited by radiation from the grating. We are adopting such an approach for our cryogenic mm-wave instrument (see Section 5), with the angle of the feeds a function of frequency.

\subsection{Numerical Evaluation of Spectrometer Designs}

In order to evaluate potential spectrometer designs, we have developed a numerical simulation which models the performance of the grating spectrometer once its facets are positioned. The model treats the EM field in a scalar approximation and considers only two dimensions, and it does not involve the details of the facet shape. Light is injected into the spectrometer at the entrance position assuming a uniform field distribution across the aperture of the entrance horn with width $w_{0}$. At the grating, the field strength at each facet is given by the diffraction pattern from this aperture. The Fraunhofer diffraction pattern in two dimensions then gives the field at any position on the grating ${ }^{27}$ :

$$
E_{i}=E_{0} w_{0} \frac{1+\cos \theta_{i}}{2 \sqrt{\lambda d_{i}}} \operatorname{sinc}\left[\frac{\pi w \sin \theta_{i}}{\lambda}\right] e^{i 2 \pi d / \lambda}
$$

where $d_{i}$ is the distance and $\theta_{i}$ is the angle from the input waveguide to the facet relative to the input waveguide axis. As each facet intercepts this field across its surface, it reradiates in a manner similar to Eq. (9). The total field at a position on the output surface is then the sum of the contributions from all facets:

$$
E_{\text {out }}=\sum_{i} \frac{E_{i} w_{i}}{\sqrt{\lambda d_{i, \text { out }}}} e^{i 2 \pi d_{i, \text { out }} / \lambda} \eta_{\text {blaze }}\left(i, \theta_{i}, \theta_{i, \text { out }}\right),
$$

where $\eta_{\text {blaze }}$ accounts for the blaze efficiency, a function of the wavelength and facet spacing and incident and diffracted angles relative to the local grating normal. To ensure that each facet has the correct contribution to the total field, $w_{i}$ is taken to be the same as the projected width of the facet as viewed from the input position, that is $w_{i}=d_{i} \delta \theta_{i}$. Then we have, for a monochromatic source diffracting from the grating:

$$
E_{\text {out }}=E_{0} w_{0} \sum_{i} \frac{1+\cos \theta_{i}}{2 \lambda \sqrt{d_{i} d_{i, \text { out }}}} d_{i} \delta \theta_{i} \operatorname{sinc}\left[\frac{\pi w \sin \theta_{i}}{\lambda}\right] \eta_{\text {blaze }}\left(i, \theta_{i}, \theta_{i, \text { out }}\right) e^{2 \pi i\left(d_{i}+d_{i, \text { out }}\right) / \lambda},
$$

and an intensity with units of power per unit length at the focal curve can be computed from $I \propto E^{*} E$. To ensure that our far-field numerical formula is not a limitation, we include contributions from several positions across the input waveguide width. By performing this calculation over an array of output locations, the spectral profile of the system can be evaluated at several frequencies, and these calculations provide the design feedback by which the overall geometry, two stigmatic frequencies, and input waveguide width are chosen.

In our numerical calculations the blaze efficiency is taken to be unity. While it is possible to make blaze efficiency calculations in a scalar approximation, such calculations are not accurate for free-space gratings and there is no reason to expect that they would be accurate for our waveguide application. Instead we rely on vector EM calculations possible with commercial software and published in for example Loewen \& Popov (1997) ${ }^{28}$ for infinite plane gratings in free space. Because the electric field is orthogonal to what would be the invariant dimension in a 2-d grating, the geometry is analogous to the TM or S polarization in standard diffraction grating terminology. Based on these published calculations and measurements, a blaze angle of $29^{\circ}$ was chosen to provide good performance over the full band. 

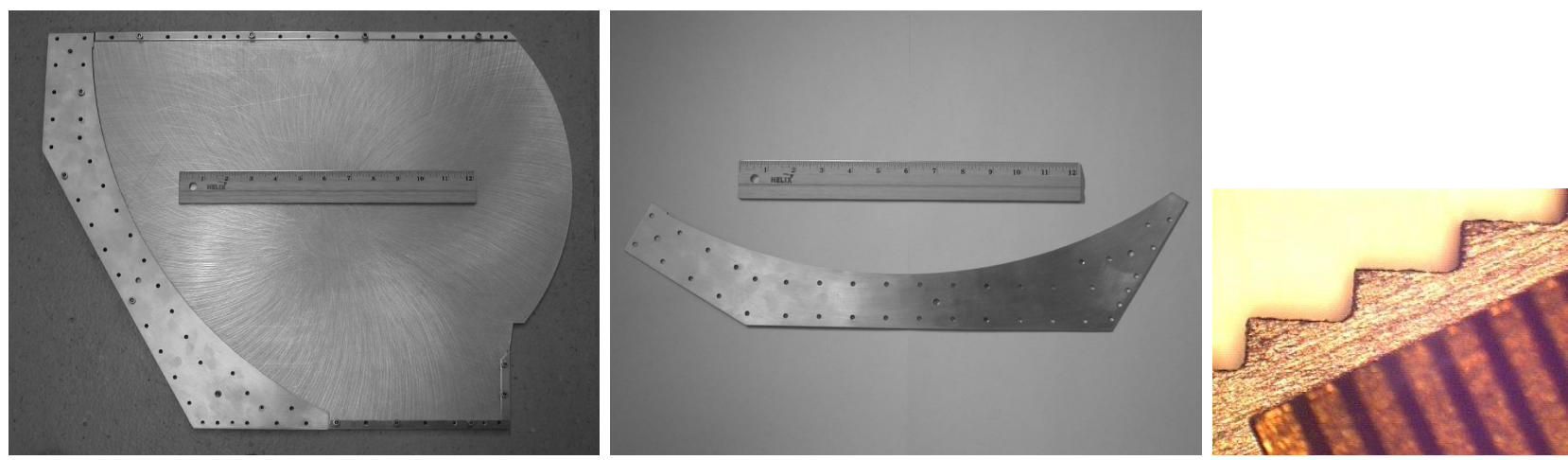

Figure 4. Photographs of first WaFIRS prototype. [Left] Spectrometer with one plate removed (before polishing). The grating is on the left, the focal curve is on the right. In this view the input location at the lower right edge of the focal curve. [Center] a view of the grating insert piece. This piece has thickness equal to the place spacing, $2.5 \mathrm{~mm}$, and the 400 facets on the top edge were machined with an wire electric discharge machine. [Right] Close-up view of the grating facets.

\section{MILLIMETER-WAVE PROTOTYPE}

We have designed and built a prototype WaFIRS module for wavelengths of 1.0-1.6 mm. For this module, the resolving power $\left(\nu / \Delta \nu_{\mathrm{FWHM}}\right)$ is more than 200 and the overall size is only $56 \mathrm{~cm} \times 42 \mathrm{~cm} \times 2.5 \mathrm{~cm}$. The grating has 400 facets, and the resulting grating curve has a length of $51 \mathrm{~cm}$. For the testing of this prototype, we have chosen an input waveguide $3.5 \mathrm{~mm}$ wide which illuminates the grating with amplitude FWHM $=150(200)$ facets at $1.0 \mathrm{~mm}(1.6 \mathrm{~mm})$. A photograph of the prototype is shown in Figure 4, with one parallel plate section removed to show the grating facets. The prototype has been tested using a backward wave oscillator (BWO) as a coherent sweep-able millimeter-wave source. A square-law diode detector is placed behind a single-mode feed to measure the transmitted power. In order to measure both the total coupling and the shape of the spectral profile, we employed two sizes of output feed size. The larger size feed is $3.2 \mathrm{~mm}$ wide, approximately matched to the spectrometer resolution element. This gives a good lower-limit to the total coupling achievable with a single-mode feed. A narrow width feed $(0.4 \mathrm{~mm})$ is also used. If fixed in a given position on the focal curve, this provides an estimate of spectral profile as frequency is scanned, though subject to an angular misalignment (see Section 4.2). With this estimate of the shape, the coupling to the large single-mode feed can be calculated and divided out to determine the true coupling of the grating and propagation medium. These results are presented in Figure 5. After the correction for the single mode measurement is applied, the results are very close to the predicted values at all but the highest frequencies. The degradation in performance at the highest frequencies probably represents a breakdown in the applicability of blaze calculations for flat gratings to our device. We are currently constructing additional gratings with different blaze angles to understand and correct the effect.

\section{SCALING TO SHORTER WAVELENGTHS}

In addition to our $1 \mathrm{~mm}$ design which is being tested, we have designed WaFIRS modules for shorter wavelengths. For the same size spectrometer, more facets are available and higher resolving power can be obtained. This scalability is a key advantage of the stigmatic design process. Of course, at some point, because the grating is only a single optical element, aberrations are inevitable at frequencies far from the stigmatic frequencies. When the physical size of the resolution element is reduced to the size of the aberrations, the scaling breaks down, and this is predicted with our numerical models. We have designed modules with the same size and shape as our $1 \mathrm{~mm}$ system for $100 \mu \mathrm{m}$ which provide resolving power between 1000 and 1600 over the same $60 \%$ bandwidth, as shown in Table 2. To implement such a system will much more technically challening - it would require holding $\sim \lambda / 10$ tolerances in the fabrication of the grating. This is not trivial since the grating is large and is free-machined rather than ruled like a traditional grating. Also, to maintain a uniform dispersion relation in the waveguide, the plates must be stiff against flexure to maintain their flat figure. 

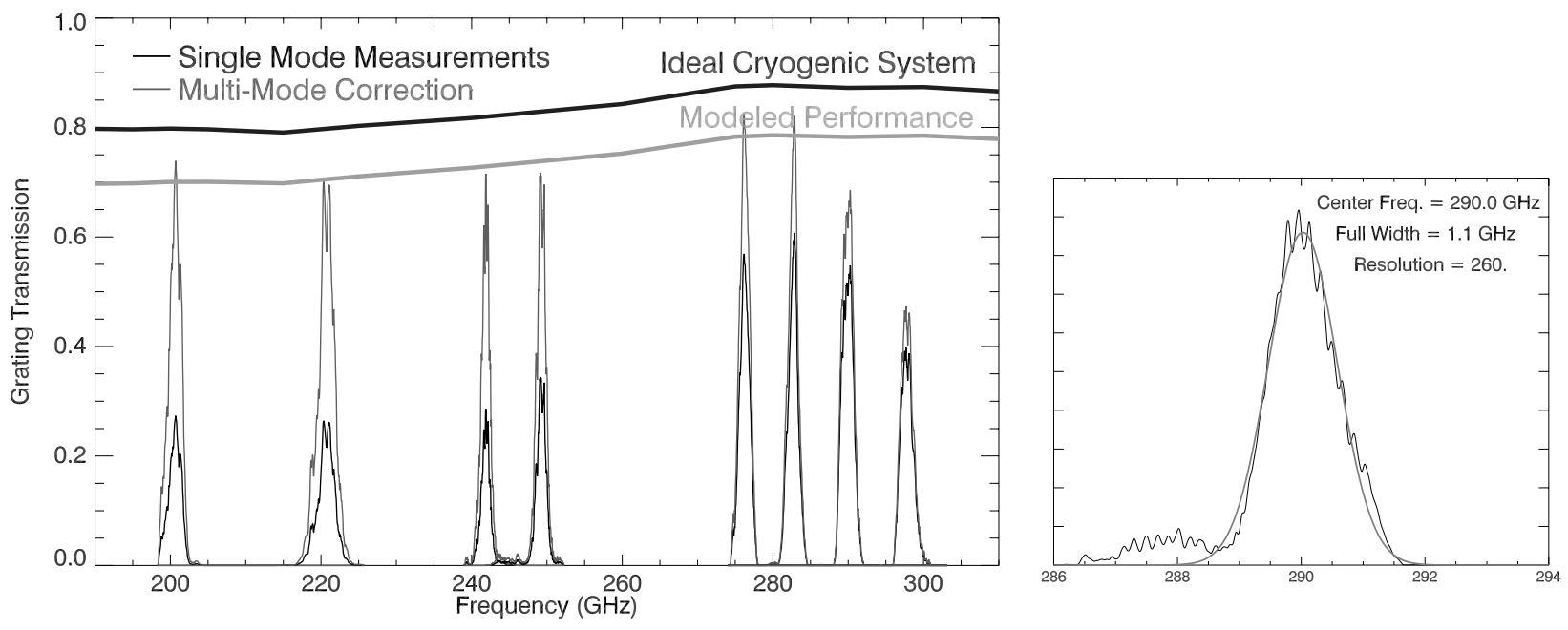

Figure 5. Verification of the WaFIRS 1.0-1.6 mm prototype. [Left] Measurements were made with a square-law detector behind a single-mode feed oriented non-optimally, which does not couple all the power in the profile. Accounting for this couping inefficiency produces the corrected curves which are close to the values expected given the waveguide propagation loss and spillover loss. The high-frequency degradation is under investigation; it is likely a blaze inefficiency which will be corrected in future designs. [Right] Measured WaFIRS prototype spectral resolution. Our present measurement apparatus uses only a single-mode feed which is oriented radially on the focal curve. For this reason, the spectral resolution can only be accurately measured near the high-frequency end of the band, where the focal curve is oriented toward the center of the grating. To within the measurement uncertainties, this is equal to the resolution predicted by the numerical model.

Table 2. WaFIRS Module Parameters

\begin{tabular}{|cccc|}
\hline Design Parameter & First Prototype & Z-SPEC Module & Far-IR Design \\
\hline Frequency Range [GHz] & $195-310$ & $195-310$ & $1900-3000$ \\
Stigmatic Frequencies [GHz] & 273,204 & 296,199 & 2800,2000 \\
Number of Detectors & & 160 & $\sim 500$ \\
Number of Facets & 400 & 480 & 4000 \\
Resolving Power & $180-250$ & $250-400$ & $1000-1600$ \\
Plate Spacing & $2.5 \mathrm{~mm}$ & $2.5 \mathrm{~mm}$ & $0.6 \mathrm{~mm}$ \\
Spacing Tolerance & $0.08 \mathrm{~mm}$ & $0.04 \mathrm{~mm}$ & $0.005 \mathrm{~mm}$ \\
Longest Dimension & $50 \mathrm{~cm}$ & $61 \mathrm{~cm}$ & $55 \mathrm{~cm}$ \\
Illumination Efficiency & $0.87-0.91$ & $0.78-0.85$ & $0.90-0.93$ \\
\hline
\end{tabular}

\section{Z-SPEC: 1 MM ASTRONOMICAL TESTBED}

To prove the WaFIRS spectrometer concept in a scientific context, we are building Z-SPEC for ground-based redshift surveys at $\lambda=1 \mathrm{~mm}^{29}$. To achieve background-limited sensitivity, we are using $160 \mathrm{Si}_{3} \mathrm{~N}_{4}$ micro-mesh bolometers ${ }^{30}$, and the entire spectrometer module is cooled to $100 \mathrm{mK}$. The parameters of the spectrometer are listed in Table 2. Light from the telescope is coupled into the spectrometer with a corrugated feed horn followed by a transformer which excites the $\mathrm{TE}_{10}$ mode in rectangular waveguide. Thermal radiation in the mid and far-IR are eliminated with low-pass mesh interference filters, while frequencies below the band are rejected by the waveguide cutoff before the input waveguide tapers up to the full plate spacing height.

A key technical challenge of this instrument is the illumination of the bolometers, which are individual devices, each consisting of a single absorber suspended with thin legs. Because of this, the absorbers cannot be 
packed with unit filling factor in the focal curve. To efficiently illuminate the detectors, we have engineering a system of alternating waveguide feeds which direct the radiation up for one detector and down for an the adjacent detector. With this scheme no tapering is required in the feed and all excited modes can propagate to the absorber, yet there is a factor of two space overhead for each detector to accommodates the frame and support legs. Our HFSS design calculations indicate that both mitered and curved waveguide bends will work well-we are building and testing prototypes of each. The feeds are consolidated in blocks of 20, each block assembled by stacking layers one waveguide-width thick. 10 detectors are then mounted on the top and bottom of each block, each in an individual removable package.

\section{SUMMARY}

To address the growing need for sensitive, broad-band spectral coverage at long wavelengths, we are developing a new architecture for far-IR / mm spectroscopy. WaFIRS uses parallel plate waveguide and curved diffraction grating and is several times more compact than conventional grating systems. Initial testing of the first prototype built for $1 \mathrm{~mm}$ is encouraging. We are constructing a cryogenic version for 200-300 GHz to be used on groundbased $\mathrm{mm}$ / submm telescopes for measuring submillimeter galaxy redshifts.

\section{ACKNOWLEDGMENTS}

We are grateful to Peter Siegel at JPL for his generous help with the backward wave oscillator. We would like to acknowledge support from the JPL Director's R\&D Fund, sponsored by NASA. JG also acknowledges NASA (NAG5-11911) and the Research Corporation (RI 0928). CMB is supported in part with a Millikan Fellowship at CIT.

\section{References}

[1] A.W. Blain, I. Smail, R.J. Ivison, and J.-P. Kneib, "The history of star formation in dusty galaxies", Mon. Not. Royal. Astron. Soc.302, pp. 632-648. 1999

[2] A.W. Blain, I. Smail, R.J. Ivison, J.-P. Kneib, and D.T. Frayer, "Submillimeter Galaxies", Physics Reports, in press. 2002

[3] G.J. Stacey, A. Poglitsch, N. Geis, M. Haggerty, J. Jackson, M. Rumitz, R. Genzel, and C.H. Townes, "The $158 \mu \mathrm{m}$ [CII] line, a measure of global star formation activity in galaxies", Astrophys. J., 373, pp. 423-433. 1991

[4] S.D. Lord, D.J. Hollenbach, M.R. Haas, R.H. Rubin, S.W.J. Colgan, E.F. Erickson, "Interstellar properties of a dual nuclear starburst: far-infrared spectroscopy of M82", Astrophys. J., 465, pp. 703-711. 1996

[5] J.W. Colbert et al., "ISO LWS spectroscopy of M82: a unified evolutionary model", Astrophys. J., 511, pp. 721-730. 1999

[6] P. Carral, D.J. Hollenbach, S.D. Lord, S.W.J. Colgan, M.R. Haas, R.H. Rubin, E.F. Erickson, "The interstellar medium in the starburst regions of NGC 253 and NGC 3256", Astrophys. J., 423, pp. 233-245. 1994

[7] S. Malhotra et al., "Far-infrared spectroscopy of normal galaxies: physical conditions in the interstellar gas", Astrophys. J., 561, pp. 766-774. 2001

[8] R. Genzel et al., "What powers ultraluminous IRAS galaxies", Astrophys. J., 493, pp. 650-662. 1998

[9] D.T. Jaffe, A.I. Harris, and R. Genzel, "Warm dense gas in luminous protostellar regions: a submillimeter and far-infrared CO line study", Astrophys. J., 316, pp. 231-242. 1987

[10] A. Harris, R.E. Hills, J. Stutzki, U.U. Graf, A.P.G. Russell, and R. Genzel, "First observations of the CO J=6-5 transition in starburst galaxies", Astrophys. J., 382, pp. L75-L79. 1991 
[11] C.M. Bradford et al., "CO J=7-6 observations of NGC 253: warm molecular gas in the starburst nucleus", Astrophys. J., in press. 2002

[12] C.M. Bradford et al., "ISO spectroscopy of OH in the starburst galaxy NGC 253", The Universe as Seen by ISO, ESA SP-427, pp, 861-864. 1999

[13] J. Fischer et al., "ISO far-IR spectroscopy of IR-bright galaxies and ULIRGs", Astrophys 83 Space Sci., 266, pp. 91-98. 1999

[14] M. Tegmark, J. Silk, M.J. Rees, A. Blanchard, T. Abel, and F. Palla, "How small were the first cosmological objects?", Astrophys. J., 474, pp. 1-12. 1997

[15] T.M. Fuller, and H.M.P. Couchman, "Simulation of primordial object formation", Astrophys. J., 544, pp. 6-20. 2000

[16] Q.D. Tran et al., "ISOCAM-CVF 5-12 $\mu \mathrm{m}$ spectroscopy of ultraluminous infrared galaxies", Astrophys. J., 522, pp. 527-543. 2001

[17] N.M. Förster Schreiber, R. Genzel, D. Lutz, D. Kunze and A. Sternberg, "Near infrared integral field spectroscopy and mid-infrared spectroscopy of the starburst galaxy M82", Astrophys. J., 552, pp. 544-571. 2001

[18] M.L. Luhman et al., "Infrared space observatory meaurements of a [C II] $158 \mu \mathrm{m}$ line deficit in ultraluminous infrared galaxies", Astrophys. J., 504, pp. L11-L14. 1998

[19] J. Stutzki et al., "Atomic carbon in M82: physical conditions derived from simultaneous observations of the [C I] fine structure submillimeter wave transitions", Astrophys. J., 477, P. L33-L37. 1997

[20] M. Gerin and T. Phillips, "Atomic carbon in galaxies", Astrophys. J., 509, pp. L17-L20. 1998

[21] W. Wild, A.I. Harris, A. Eckart, R. Genzel, U.U. Graf, J.M. Jackson, A.P.G. Russell, and J. Stutzki, "A multi-line study of the molecular interstellar medium in M82's starburst nucleus", Astron. Astrophys., 265, pp. 447-464. 1992

[22] D.J. Schlegel, D.P. Finkbeiner, and M. Davis, "Maps of dust infrared emission for use in estimation of reddening and cosmic microwave background radiation foregrounds", Astrophys. J., 500, pp. 525-553. 1998

[23] H.A Rowland, "On concave gratings for optical purposes", Phil. Mag., 16, pp. 197-210. 1883

[24] M. Born and E. Wolf, "Principles of Optics", Cambridge: Cambridge U. Press. 1999

[25] D.M. Pozar, "Microwave Engineering", New York: John Wiley \& Sons. 1998

[26] K.A. McGreer, "Tunable planar concave grating demultiplexer", IEEE Phot. Tech. Let., 8, pp. 551-553. 1996

[27] A. Papoulis, "Systems and transforms with applications in optics", New York: McGraw-Hill. 1968

[28] E.G. Loewen and E. Popov, "Diffraction gratings and applications", New York: Marcel Dekker. 1997

[29] B. Naylor et al., "Z-Spec, a broadband direct-detection millimeter-wave spectrometer", Proc SPIE, 4855-68. 2002

[30] A. Turner et al. "Silicon nitride micromesh bolometer array for submillimeter astrophysics", Applied Optics, 40, pp. 4921-4932. 2001 